\title{
A Mathematical Study of Sample Modulation at a Membrane Inlet Mass Spectrometer-Potential Application in Analysis of Mixtures
}

\author{
Fei L. Overney and Christie G. Enke \\ Department of Chemistry, The University of New Mexico, Albuquerque, New Mexico, USA
}

\begin{abstract}
An ANSI $C$ program that simulates the diffusion profiles of sample modulation at a membrane inlet system has been developed to study the characteristics of modulated diffusion profiles. The program produces concentration profiles within the membrane and flux values at the exit side of the membrane as a function of time. Sample concentration on the inlet side can be switched between zero and an arbitrary value with a square or asymmetric cycle. Achievement of steady-state diffusion between alternations is not required. With this computer simulation, the flux profiles of analytes through a membrane inlet have been studied as a function of diffusion coefficient, modulation frequency, and concentration. The amplitude, shape, and time lag or phase angle of the flux profile are shown to be related directly to analyte concentration and diffusivity. A method that involves a set of linear equations is proposed to resolve mixtures of diffusing analytes based on differences in the time dependence of their flux profiles. (J Am Soc Mass Spectrom 1996, 7, 93-100)
\end{abstract}

$\mathrm{T}$ he direct analysis of mixtures by mass spectrometry depends upon principal component analysis to determine the sample components that contributed to the mixture spectrum. This method relies on constant response factors for each component and is limited in sample complexity and dynamic range. To obtain additional data for component resolution, discrimination along the time axis by (1) componentspecific time-dependent response and (2) individual intensity time profiles for each mass-to-charge ratio can be used. A commonly employed example of this approach is gas chromatography-mass spectrometry (GC/MS). Chromatography provides different response time profiles for nearly all components, but the time between successive determinations of sample composition cannot be less than the longest component retention time. To provide more frequent updates on samples with varying composition, we are exploring the use of a membrane inlet with modulated sample introduction.

A majority of the membrane inlet work or membrane introduction mass spectrometry (MIMS) to date has employed steady-state diffusion through membranes [1-4]. MIMS is applied to selectively transport analytes that bear particular functional groups and enrich them relative to the inlet solution [5]. Tsai et al. [6] have made a theoretical analysis of flow injection MIMS. They modeled the dynamics of the flow cell

Address reprint requests to Professor Christie G. Enke, Department of Chemistry, The University of New Mexico, Albuquerque, NM 87131. volume and membrane permeation to obtain analyte flux profiles for a variety of inlet concentration profiles that all return to zero flux between repetitions.

We report here on the results of our mathematical simulation of the modulated diffusion process. The sample stream modulation is provided by an alternating valve that opens and closes periodically. We have ignored mixing on the sample side of the membrane because our experimental system uses a tubular membrane with negligible dead time and dead volume. A time-dependent response pattern, called a chronogram, is produced for the ions of each mass-to-charge ratio in the mixture spectrum. The shapes and amplitudes of the chronograms depend on the concentrations and diffusivities of the mixture components.

We have developed a computer program to simulate the Fickian diffusion process (which obeys Fick's first and second laws) through a membrane with a modulated sample stream. We have chosen a finite difference method [7] for its simplicity and expandability. Specifically, the Crank-Nicolson method $[8,9]$ has been applied to solve the corresponding parabolic partial differential equation numerically. In our simulation, the time dependence of the analyte concentration caused by the alternating valve is modeled as or close to a periodic step function. The resulting diffusion flux profile at the mass spectrometer inlet converges to a complex periodic function that has the same frequency as the analyte modulation, but delayed in time by the diffusion process. The wave shape and time delay of the analyte diffusion flux depend upon the diffusion 
coefficient of the analyte through the membrane. The amplitude of the flux variations is proportional to the analyte concentration. The acquisition of the chronogram for each mass-to-charge ratio in the mass spectrum will provide important additional data for mixture analysis.

The periodic variation in the flux of the analyte into the mass spectrometer causes related periodic intensity variations for the mass-to-charge ratios related to this analyte. From these mass-to-charge ratio chronograms, the concentrations and diffusivities of the analytes can be determined. For mixture analysis, the chronogram of each mass-to-charge ratio channel is contributed to by all the sample components that are represented in that mass-to-charge ratio channel. The concentrations of all the components can be calculated from the chronogram data for all mass-to-charge ratio channels by solving a set of linear equations.

\section{Theory}

In general, membrane processes are comprised of the membrane, the feed stream (sample), the reject stream (waste or vent), and the permeate stream (sample extract). The permeate stream is enriched in analytes due to the selective permeation properties of the membrane. The permeation of an analyte through a membrane involves three processes:

1. Selective partitioning of the analyte from the sample into the membrane polymer matrix.

2. Selective diffusion of the analyte through the membrane.

3. Desorption of the analyte from the membrane into a vacuum or sweep gas [10].

The mathematical analysis of the sample concentration in the permeate stream is developed subsequently for both steady-state and modulated sample introduction.

\section{Analysis of Steady State Permeate Stream (Membrane Inlet Mass Spectrometry)}

A membrane inlet is generally used to provide analyte enrichment in the sample matrix. From the perspective of mass spectrometry, the steady-state diffusion of the mixture provides a mixture spectrum for which a principal component analysis is then used to resolve each component.

Diffusion through the membrane is assumed to be the rate determining process, whereas partition at the sample surface and desorption from the permeate surface are considered to be instantaneous [11, 12].

Steady-state permeation is described by Fick's first law:

$$
\text { flow }_{\mathrm{st}}=-A D(\partial C / \partial x)_{x=d}
$$

where flow $_{\text {st }}$ is the steady-state flow rate or permeation rate (flux times membrane area) of a substance in the permeate, $A$ is the surface area of the membrane,

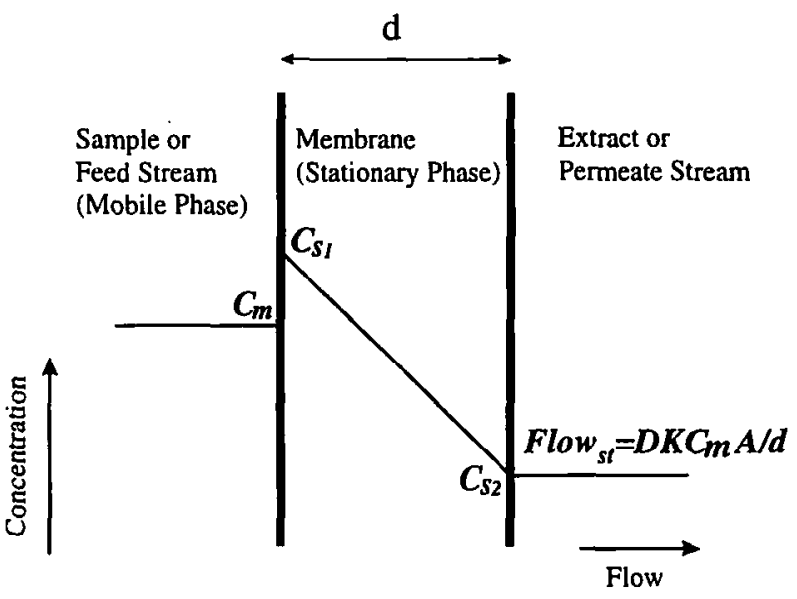

Figure 1. The components of the permeation process for a sheet membrane. $C_{m}$ is the concentration in the sample stream, $C_{51}$ and $\mathrm{C}_{\mathrm{S}_{2}}$ are the surface concentrations at membrane inlet and outlet respectively. Flow ${ }_{\mathrm{st}}$ is the steady-state flow rate.

$D$ is the diffusion coefficient of the substance in the membrane polymer, and $(\partial C / \partial x)$ is the concentration gradient at the point in the membrane for which the diffusion rate is being calculated (generally at the exit surface). For the diffusion through a sheet membrane at steady state, as shown in Figure $1,(\partial C / \partial x)$ is constant through the membrane and eq 1 gives

$$
\text { flow }_{\mathrm{st}}=A D\left(C_{\mathrm{S} 1}-C_{\mathrm{S} 2}\right) / d \quad \text { (sheet membrane) }
$$

For a hollow fiber membrane at steady state [10], it follows that

$$
\begin{aligned}
\text { flow }_{\mathrm{st}}= & 2 \pi L D\left(C_{\mathrm{S} 1}-C_{\mathrm{S} 2}\right) / \ln \left(d_{\mathrm{o}} / d_{\mathrm{i}}\right) \\
& \text { (hollow fiber membrane) }
\end{aligned}
$$

where $C_{S 1}$ and $C_{S 2}$ are the concentrations of the substance at the feed surface and at the permeate surface of the membrane, respectively, $d$ is the thickness of the sheet membrane, $L$ is the length of the hollow fiber membrane, and $d_{\mathrm{o}}$ and $d_{\mathrm{i}}$ are the outer and inner diameters of the hollow fiber, respectively.

If the permeate side of the membrane is exposed to the vacuum or swept with a carrier gas, a concentration gradient is established and $C_{\mathrm{S} 2}$ becomes very small relative to $C_{\mathrm{S} 1}$ and therefore $C_{\mathrm{S} 2}$ can be considered to be equal to zero. This concentration gradient is the driving force for diffusion. The concentration $C_{\mathrm{S} 1}$ is established by the partitioning process, for which the distribution coefficient $K$ is given by $K=a_{\mathrm{s}} / a_{\mathrm{m}} \cong$ $C_{\mathrm{s} 1} / C_{m}$, where $a_{\mathrm{s}}$ and $a_{\mathrm{m}}$ are the activities of the substance in the stationary and mobile phase and $C_{m}$ is the concentration of the substance in the feed (mobile phase) [10]. Equation 2 may be rewritten for the sheet membrane as

$$
\text { flow } \left._{\mathrm{st}}=A D K C_{m} / d \quad \text { (sheet membrane } C_{\mathrm{S} 2}=0\right)
$$

Equation 3 for the hollow fiber is given by

$$
\begin{aligned}
\text { flow }_{\mathrm{st}}= & 2 \pi L D K C_{m} / \ln \left(d_{\mathrm{o}} / d_{\mathrm{i}}\right) \\
& \left(\text { fiber membrane } C_{\mathrm{S} 2}=0\right)
\end{aligned}
$$


Equations 4 and 5 can be applied to both gas and aqueous samples [13]. At a given temperature and pressure, the diffusivity (or diffusion coefficient) and the dimension factor $[A / d$ for the sheet or $2 \pi L / \ln \left(d_{\mathrm{o}} / d_{\mathrm{i}}\right)$ for the hollow fiber] are constants. The flow rate and therefore the analytical signal $I_{i}$ is directly proportional to the sample concentration such that

$$
I_{i}=Q_{i} \text { flow }_{i}=\mathrm{rf}_{i} C_{m,}
$$

where $Q_{i}$ is the absolute instrumental response factor and $\mathrm{rf}_{i}$ is the analytical response factor for compound $i$.

\section{Analysis by Modulated Sample Introduction (Modulated Membrane Inlet Mass Spectrometry)}

The sample modulation is controlled by an alternating valve (as shown in Figure 2) that periodically switches between the sample stream and the background stream. This will cause a periodic variation in the analyte flux with a frequency equal to the sample modulation frequency. The magnitude, time lag, and wave shape of the analyte flux are functions of the analyte's concentration and diffusivity.

Non-steady-state permeation is governed by Fick's second law:

$$
\partial C / \partial t=-D\left(\partial C^{2} / \partial x^{2}\right)
$$

There are two situations with regard to modulated sample introduction: (1) alternation between two inlet conditions with period long enough for the analytes of interest to reach steady-state diffusion in each half cycle and (2) alternation with a faster switching period during which the analytes do not achieve steady-state diffusion.

Switching between steady-state conditions. Switching between steady-state conditions provides two special

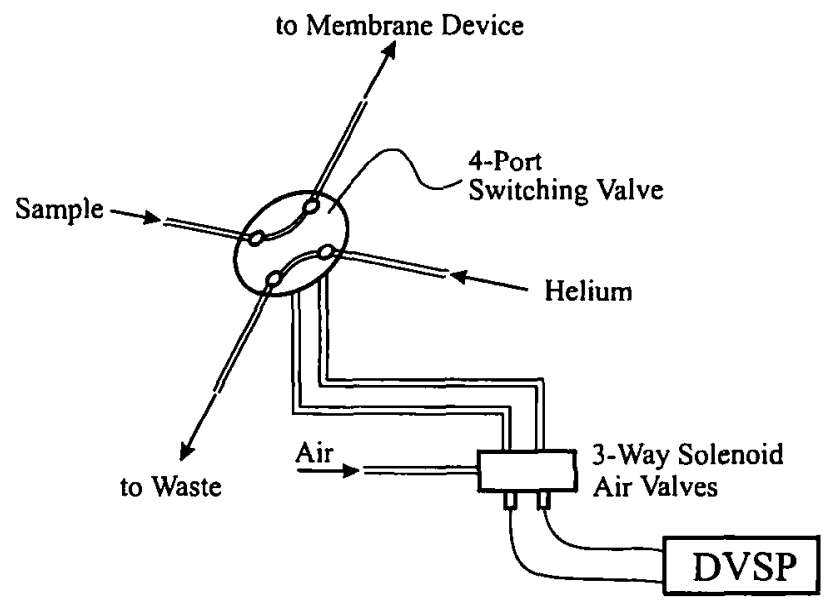

Figure 2. The modulation of sample stream is accomplished by a four-port switching valve driven by an air actuator. The timing of the alternation between sample and helium flow to the membrane device is controlled by the digital valve sequence programmer (DVSP). boundary conditions: one when the alternating valve is turned on, which produces the rising edge of the permeate flux, and one when the valve is turned off, which causes the falling edge of the permeate flux. Under both conditions Fick's second law can be solved analytically to provide equations for the flux as a function of time.

CONDITION I. For the rising edge, the boundary conditions are

$$
\begin{array}{ll}
C=0, & t=0 \\
C=C_{\mathrm{S} 1}, & x=0 \\
C=0, & x=d
\end{array}
$$

The mathematical solution for the time-dependent rate of a diffusion process through a flat sheet membrane with thickness $d$ that follows a step change in sample concentration (the typical permeation response curve) is [14]

$$
\begin{aligned}
F(D, d, l)_{\text {norm }} & =F(D, d, t) / F_{\mathrm{st}} \\
& =1+2 \sum_{n=1}^{x}(-1)^{n} \exp \left[-(n \pi / d)^{2} D t\right]
\end{aligned}
$$

where the $F$ and $F_{\text {st }}$ indicate the flux (flow per centimeter squared) at time $t$ and at steady-state conditions, respectively.

Condition II. For the falling edge, the boundary conditions are

$$
\begin{array}{ll}
C=0, \quad x=0, & t \geq 0 \\
C=0, \quad x=d, & t \geq 0 \\
C=C_{S 1}(1-x / d), & 0<x<d, \quad t=0
\end{array}
$$

The concentration profile inside the membrane is [8]

$$
\begin{aligned}
C= & \frac{2}{d} \sum_{n=1}^{\infty} \sin \frac{n \pi x}{d} \exp \left[-\left(\frac{n \pi}{d}\right)^{2} D t\right] \int_{0}^{d} C_{\mathrm{S} 1}\left(1-\frac{x^{\prime}}{d}\right) \\
& \times \sin \left(\frac{n \pi x^{\prime}}{d}\right) d x^{\prime}
\end{aligned}
$$

The definite integral gives a constant $C_{s 1} / n \pi$. Equation 11 can be rewritten as

$$
C=\frac{2 C_{\mathrm{S} 1}}{\pi} \sum_{n=1}^{\infty} \frac{1}{n} \sin \left(\frac{n \pi x}{d}\right) \exp \left[-\left(\frac{n \pi}{d}\right)^{2} D t\right]
$$

Because flux of the surface $x=d$ is $F(t)=-(D$ $\partial C / \partial x)_{x=d}$, the normalized flux expression can be readily derived as

$$
\begin{aligned}
F(D, d, t)_{\text {norm }} & =F(D, d, t) / F_{\text {st }} \\
& =-2 \sum_{n=1}^{x}(-1)^{n} \exp \left[-(n \pi / d)^{2} D t\right]
\end{aligned}
$$

Figure 3 shows the analytical results for the rising and falling edges of toluene. The simulated membrane 


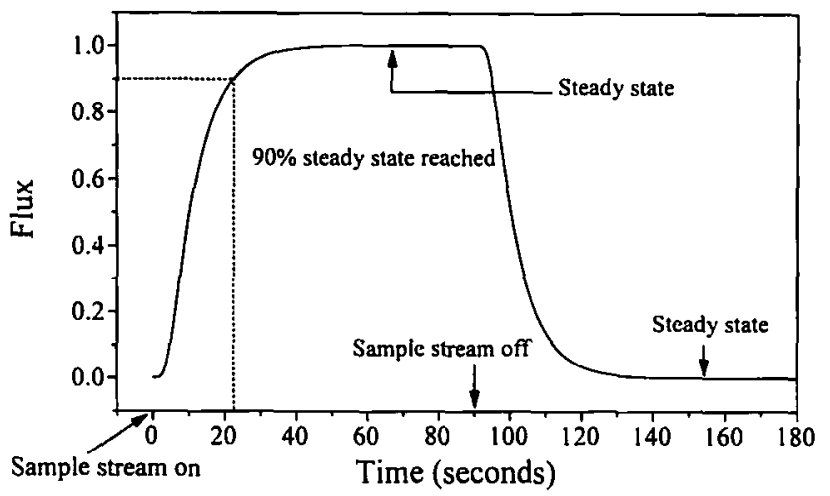

Figure 3. Simulated modulation profile of toluene to the steady-state. The flux increases until steady-state is reached with the sample stream on, then decreases to zero with the sample stream off. The value of the steady-state flux is normalized to 1.0.

thickness is $0.16 \mathrm{~mm}$. The membrane material is silicone rubber in which the diffusivity for toluene is $3.5 \times 10^{-6} \mathrm{~cm}^{2} / \mathrm{s}[10]$. As we see in this figure, it takes $22 \mathrm{~s}$ to reach $90 \%$ of the steady-state value. At $90 \mathrm{~s}$, when the sample concentration is turned off, the flux has been at $100 \%$ of the steady-state value for about 30 s. Consider sample components that may have considerably lower diffusion constants, the minimum cycle time would be several minutes if achievement of steady state were required.

Rapid modulation. To implement faster modulation, it is necessary to decrease the membrane thickness, increase the diffusion coefficient (perhaps by temperature elevation), or allow switching before the achievement of steady state. Of these options, switching prior to steady state is the most general solution and it avoids spending much of the cycle time in the less informative steady-state condition.

Because the alternating valve is switched off before steady state has been reached, the concentration profile of the analyte inside the membrane is not linear. When the alternating valve is switched back on, the concentration of the analyte inside the membrane is not zero. From a mathematical perspective, the time boundary conditions become so complex that an analytical solution of Fick's second law is impossible to obtain. Under such circumstances, a numerical analysis is required. The development of a method for a numerical analysis of this problem and evaluation of the analytical results in terms of the goals of chemical analysis are the foci of this paper.

Method for flux determination with rapid sample modulation. There are different numerical methods available to solve parabolic, partial differential equations such as the diffusion equation numerically. The three most common methods are the explicit and the implicit schemes [15] and the Crank-Nicolson (CN) scheme [9, 16]. In this work, we have applied the $\mathrm{CN}$ scheme, which is a well proven discrete sample point grid method. The $\mathrm{CN}$ method has two major advantages.
First of all, it is numerically very stable. Second, due to the use of half-time steps, high precision can be achieved.

Starting with the diffusion equation

$$
\frac{\partial C}{\partial t}=D \frac{\partial^{2} C}{\partial x^{2}}
$$

we approximate the time derivative for the concentration at half-time step $C(t+1 / 2 \delta t)$ for a given gridpoint $i$ with

$$
\begin{aligned}
\frac{\partial C_{i}(t+1 / 2 \delta t)}{\partial t} & \approx \frac{C_{i}(t+\delta t)-C_{i}(t)}{\delta t} \\
& \equiv \frac{C_{i}^{\prime}-C_{i}}{\delta t}
\end{aligned}
$$

where $C_{i} \equiv C_{i}(t)$ and $C^{\prime} \equiv C(t+\delta t)$, respectively.

Second, we approximate the second derivative

$$
\frac{\partial^{2} C_{i}}{\partial x^{2}}=\frac{C_{i-1}-2 C_{i}+C_{i+1}}{h^{2}}
$$

where $h=\delta x$, and approximate $C(t+1 / 2 \delta t)$ with

$$
C\left(t+\frac{1}{2} \delta t\right) \approx \frac{C(t)+C(t+\delta t)}{2}=\frac{C+C^{\prime}}{2}
$$

Combining eqs 16 and 17, we get a concentration approximation at the half-time step:

$$
\begin{aligned}
& \frac{\partial^{2} C_{i}(t+1 / 2 \delta t)}{\partial x^{2}} \\
& \quad \approx \frac{1}{2}\left[\frac{C_{i-1}-2 C_{i}+C_{i+1}+C_{i-1}^{\prime}-2 C_{i}^{\prime}+C_{i+1}^{\prime}}{h^{2}}\right]
\end{aligned}
$$

Since

$$
\frac{\partial C_{i}(t+1 / 2 \delta t)}{\partial t}=D \frac{\partial^{2} C_{i}(t+1 / 2 \delta t)}{\partial x^{2}}
$$

we find that

$$
\begin{aligned}
& \frac{C_{i}^{\prime}-C_{i}}{\delta t} \\
& \quad=\frac{D}{2}\left[\frac{C_{i-1}-2 C_{i}+C_{i+1}+C_{i-1}^{\prime}-2 C_{i}^{\prime}+C_{i+1}^{\prime}}{h^{2}}\right]
\end{aligned}
$$

Third, we rearrange for all $i$ to $C_{i-1}^{\prime}+a C_{i}^{\prime}+C_{i+1}^{\prime}=b_{i}$ with

$$
\begin{aligned}
& a=-\frac{2}{\lambda}(\lambda+1) \\
& b_{i}=-C_{i-1}+\frac{2(\lambda-1)}{\lambda} C_{i}-C_{i+1} \\
& \lambda=\frac{D \delta t}{h^{2}}
\end{aligned}
$$


This is a tridiagonal system of equations

$$
\begin{aligned}
& C_{0}^{\prime}+a C_{1}^{\prime}+C_{2}^{\prime}=b_{1} \\
& C_{1}^{\prime}+a C_{2}^{\prime}+C_{3}^{\prime}=b_{2} \\
& \vdots \\
& C_{i-1}^{\prime}+a C_{i}^{\prime}+C_{i+1}^{\prime}=b_{i} \\
& C_{m-1}^{\prime}+a C_{m}^{\prime}+C_{m+1}^{\prime} \vdots \\
& b_{m}
\end{aligned}
$$

which can be solved with known values at $t=0$ and the values at the boundary $C_{0}^{\prime}$ (the concentration at inlet side of membrane) and $C_{m+1}^{\prime}$ (the concentration at outlet side of the membrane at all times) [17].

We have developed an ANSI C program and adapted some of the numerical subroutines published by Cambridge University Press [16] to resolve the concentrations at 500 discrete points inside the membrane by using 1000 time intervals for each modulation cycle. With this program, the concentrations inside the membrane can be determined progressively through time. All the $C^{\prime}$ concentrations at $t+\delta t$ can be calculated by knowing the concentrations $C$ at time $t$ for all grid points $1 \leq i \leq 500$. Concentrations at the boundaries where $x=0$ and $x=d$ are known at all times. For the initial time $t=0$, all concentrations for all grid points $i$ are known as well. Figure 4 shows the concentration profiles for a non-steady-state alternation, plotted after each 10 iterations, inside the membrane. The calculated flux profile shown in Figure 5 begins with zero analyte concentration inside the membrane and applies a switching time that is just a little too short to achieve steady-state diffusion. After several alternations, the pattern becomes exactly repetitive.

\section{Results and Discussion}

\section{Dependence of Flux Profile Parameters on Component Diffusivity}

Phase shift method. In the case where the surface concentration at the membrane inlet in the "on" state is kept constant, there is a correlation between the difu-
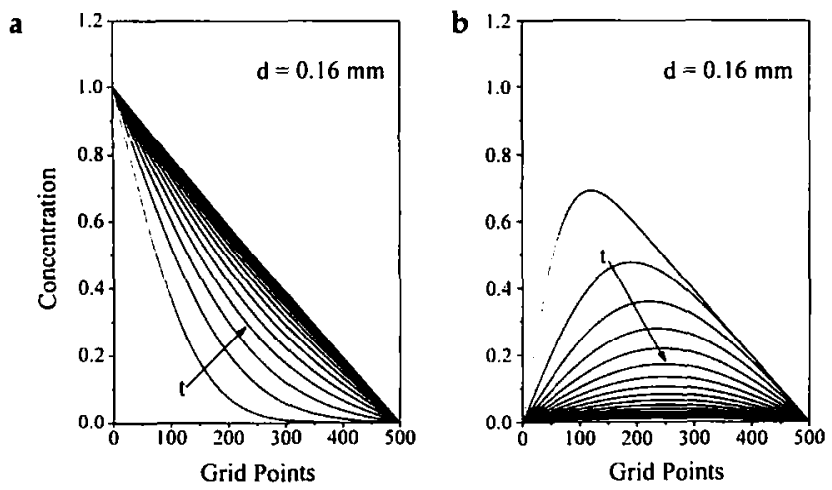

Figure 4. Calculated variation of the concentration profiles inside the membrane as function of time. The time interval between successive profiles is $6 \mathrm{~s}$ for an analyte with $D=3.5 \times$ $10^{-6} \mathrm{~cm}^{2} / \mathrm{s}$. (a) The case when the alternating valve is turned on with zero initial concentration. (b) The case when the alternating valve is turned off after achievement of steady-state diffusion.

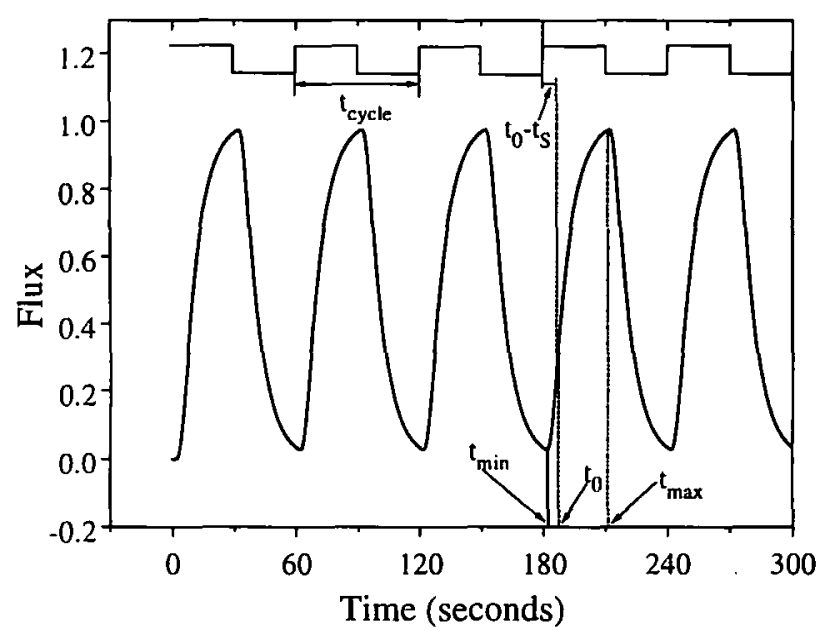

Figure 5. Normalized flux profile for the conditions: Membrane thickness $d=0.16 \mathrm{~mm}$, diffusivity $D=3.5 \times 10^{-6} \mathrm{~cm}^{2} / \mathrm{s}$. The on and off times for the sample stream are both $30 \mathrm{~s}$. The on and off of the sample stream are indicated by a step function shown below the flux profile. $t_{0}=186.83 \mathrm{~s}$, $t_{\text {cycle }}=60 \mathrm{~s}, t_{0}-t_{\mathrm{S}}=6.83$ $\mathrm{s}, t_{\min }=181.93 \mathrm{~s}$, and $t_{\max }=211.94 \mathrm{~s}$.

sivity and the phase shift $(\theta)$, which is defined as the time lag between the modulation step function and the periodical chronogram signal,

$$
\theta=f(D)=\frac{t_{0}-t_{s}}{t_{\text {cycle }}} \times 360^{\circ}
$$

where $t_{0}$ is called the inflection point. The value of $t_{0}$ is determined by plotting $d^{2} F_{i} / d t^{2}$ versus time, with $t_{0}$ being the time on the chronogram rising edge when $d^{2} F_{i} / d t^{2}$ equals zero. The time when the corresponding sample modulation step cycle starts is $t_{\mathrm{s}}$. The duration of a sample modulation cycle is $t_{\text {cycle }}$. Figure 6 indicates the correlation between $\theta$ and diffusivity. Because the relationship is monotonic, once the working curve is obtained, diffusivities can be determined.

Shape factor method. Intriguingly, we have found that the diffusivities correlate linearly with a chronogram parameter, which we define as the shape factor $\Omega$ :

$$
\Omega=k \times D
$$

where

$$
\Omega=\frac{t_{\max }-t_{\min }}{t_{0}-t_{\mathrm{S}}}
$$

The terms $t_{0}$ and $t_{\mathrm{S}}$ have the same meaning as in the phase shift method. The term $t_{\min }$ is the closest time minimum of the periodic signal (flux profile) before $t_{0}$, and $t_{\max }$ is the closest time maximum on the flux 


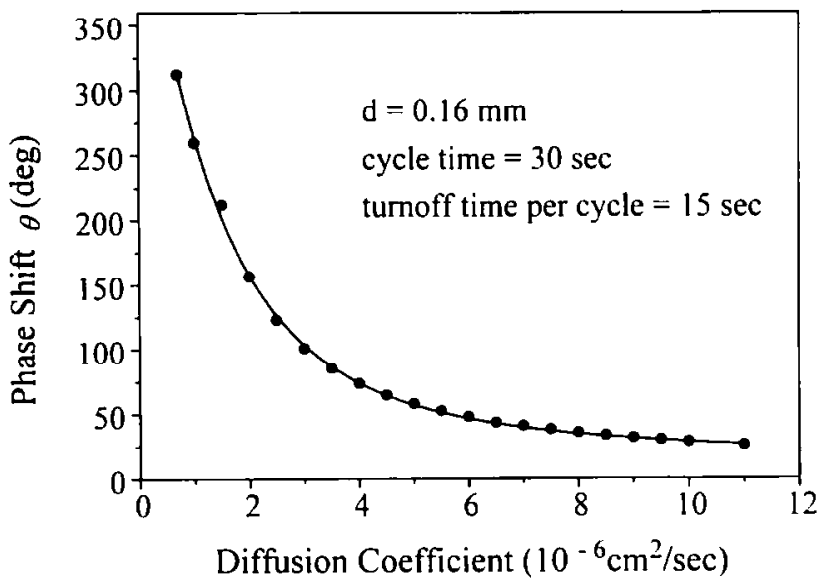

Figure 6. Relationship between phase shift and diffusivity. Dots are the values of phase shift $\theta$ obtained from the normalized flux profiles calculated for various values of $D$. The solid line was fitted with a series that contained three exponential functions.

profile after $t_{0}$. This linear relationship, as shown in Figure 7, has been demonstrated over the tenfold diffusivity range tested. As a result of the linearity between diffusivity and shape factor, the diffusivities from various analytes can be determined conveniently and verified, which makes the shape factor method easier to apply than the phase shift method over a broad diffusivity range. However, at the low diffusivity end, the phase shift method may have a greater sensitivity in diffusivity determination than the shape factor method.

\section{Flux Magnitude Related to Concentration}

We have evaluated three magnitude measures for the periodic flux profile waveform. These are the maximum value flux $\max _{\text {max }}$ equal to the maximum flux in the cycle, the peak-to-peak value flux $p_{p-p}$ equal to the difference between the maximum and minimum flux, and the midpoint value flux ${ }_{\text {mid }}$ equal to the average of the maximum and minimum values.

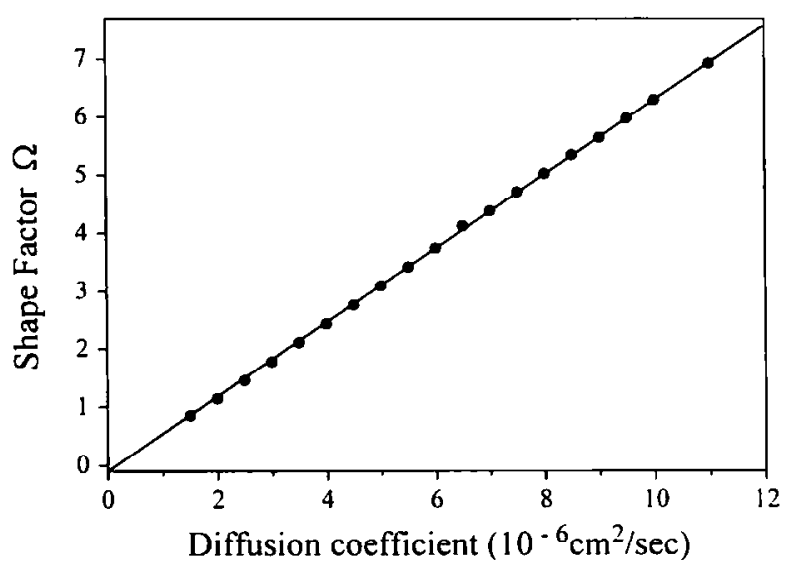

Figure 7. Diffusivity can be calculated easily from its linear correlation with the shape factor $\Omega$.

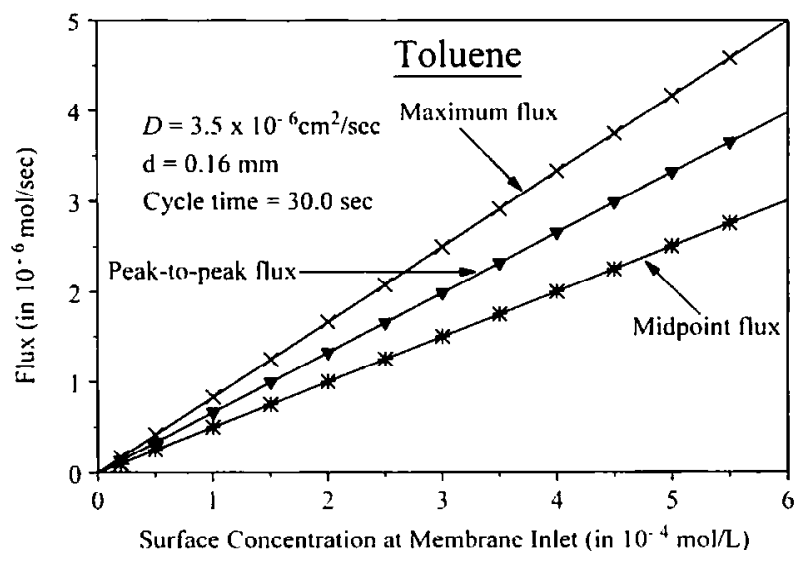

Figure 8. All flux profile magnitude measures are proportional to the analyte concentrations.

For a specific component, our simulation shows that all three measures are directly proportional to the concentration as shown in Figure 8:

$$
\begin{aligned}
& \text { flux }_{\text {max }}=k_{1} C_{\mathrm{S}} \\
& \text { flux }_{\text {mid }}=1 / 2\left(\text { flux }_{\text {max }}+\text { flux }_{\text {min }}\right)=k_{2} C_{\mathrm{S}} \\
& \text { flux }_{\mathrm{p}} \mathrm{F}=\text { flux }_{\text {max }}-\text { flux }_{\text {min }}=k_{3} \mathrm{C}_{\mathrm{S}}
\end{aligned}
$$

These results are not surprising because all measures are fractions of the steady-state flux, which is also proportional to concentration. We can assume that the partitioning process at the membrane inlet surface is a thermodynamic process on the diffusion time scale. In other words, the distribution coefficient $K=C_{s} / C_{m}$ is a constant.

\section{Flux Magnitude Related to Diffusizity}

The simulation results shown in Figure 9 indicate that with constant surface concentration, the maximum flux and the peak-to-peak amplitude of the flux profile

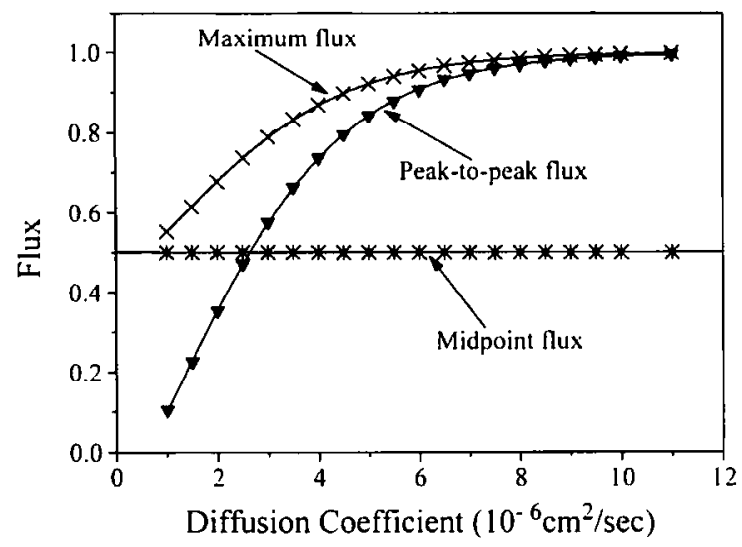

Figure 9. The maximum normalized flux and the peak-to-peak amplitude increase with diffusivity to a limiting value. The midpoint normalized flux is independent of diffusivity. 
increase with increasing diffusivity until the steadystate value is reached. On the other hand, the midpoint flux is independent of diffusivity. Due to this independence, the midpoint flux is more resistant to the various environmental changes such as temperature. As a result, it will be more accurate and precise to use the midpoint flux as a measure of sample concentration.

\section{Mixture Analysis}

For dilute mixtures of analytes, Henry's law is obeyed. At such concentrations, there are almost no interactions between analyte molecules. The "solvent" in this case is the silicon membranc. Mlthough diffusivities of all species through a membrane can be affected by significant concentrations of the analytes, we assume that at trace levels, these effects will be negligible. In this case, the mixture response will therefore exhibit simple additive properties. In other words, the total permeate rate or response signal $I_{\mathrm{T}}$ is the simple summation of the permeate rate or response signal of $N$ individual components present in the mixture at any given time.

In terms of mixture analysis, two main types of analyses are encountered. The first is general qualitative analysis where the identification and quantitation have to be accomplished simultaneously. Of greater interest in process control, where rapid updates on mixture composition are most desirable, is targeted analysis in which the identity of the mixture components is known, but their concentrations are not. In such a case, the normalized flux profile for each component $f_{i}(t)$, is

$$
f_{i}(t)=\frac{\mathrm{flux}_{i}}{\operatorname{flux}_{\mathrm{st}}}=\frac{I_{i}(t)}{\mathrm{rf}_{i} C_{m_{i}}}
$$

where $f_{i}(t)$ is only a function of time, which varies from 0 to 1 . For a given membrane setup (e.g., certain membrane thickness and material), at any given time, the normalized flux profile for a chemical compound of interest equals a particular number between 0 and 1 . We could run an experiment beforehand for each individual component to obtain its normalized flux profile. The analytical response factor for each species, denoted with $\mathrm{rf}_{i}$, can be determined by running a standard sample. As a consequence, the $N$ unknown concentrations can be resolved by solving a set of linear equations at $N$ different times:

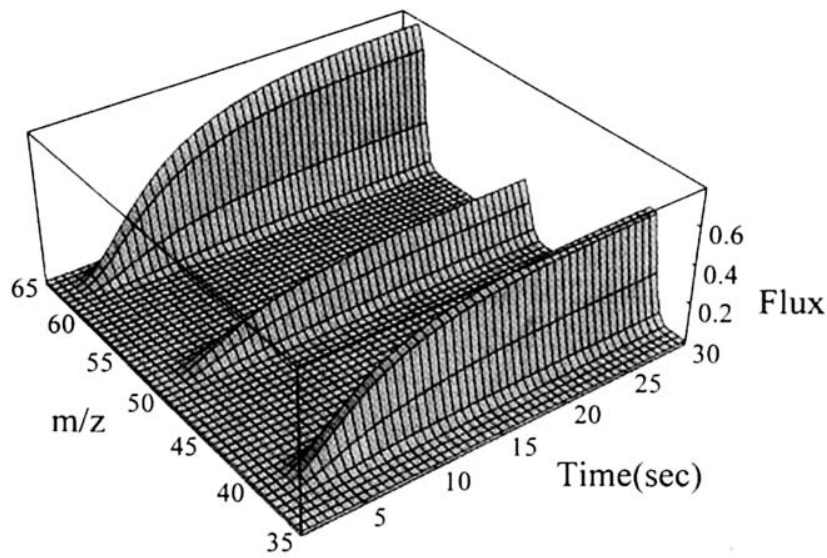

Figure 10. A three-dimensional response plot with peaks at $m / z 39,51$, and 63 for benzene (diffusivity $4.9 \times 10^{-6} \mathrm{~cm}^{2} / \mathrm{s}$ ) where the sample stream is turned on.

Notice that eq 27 is true for every mass-to-charge ratio channel that has been detected. In practice, each massto-charge ratio channel will be affected by only a subset of the total number of compounds present, so that for any given mass-to-charge ratio channel, eq 27 will have fewer than $N$ terms. Similarly, all channels affected by only a single component will have a response that follows its diffusion flux profile. Figure 10 demonstrates a three-dimensional response of a multichannel detection for a simple compound. If more than one component of the mixture affects the response at any given channel, the response curve on the plane spanned by time and mass-to-charge ratio for that channel is the summation of the individual responses, and eq 27 can be applied to determine the concentrations of these components. Usually each component of the mixture can be detected at several mass-to-charge ratio channels; therefore the quantitative analysis of each component can be made numbers of times. Clearly, the time resolution enables the multiple lines in eq 23 and will provide a critical third dimension to the response matrix for improved mass spectral analysis of mixtures.

\section{Conclusions}

For a Fickian diffusion system, with modulated membrane inlet mass spectrometry, the phase angle and shape of the individual mass-to-charge ratio chronograms affected by only single components depend on the diffusivities of the components from which they are derived. As a result, the single component diffu-

$$
\begin{aligned}
& \mathrm{rf}_{1} f_{1}\left(t_{1}\right) C_{m_{1}}+\mathrm{rf}_{2} f_{2}\left(t_{1}\right) C_{m_{2}}+\cdots+\mathrm{rf}_{N} f_{N}\left(t_{1}\right) C_{m_{N}}=I_{\mathrm{T}}\left(t_{1}\right) \\
& \mathrm{rf}_{1} f_{1}\left(t_{2}\right) C_{m_{1}}+\mathrm{rf}_{2} f_{2}\left(t_{2}\right) C_{m_{2}}+\cdots+\mathrm{rf}_{N} f_{N}\left(t_{2}\right) C_{m_{N}}=I_{\mathrm{T}}\left(t_{2}\right) \\
& \mathrm{rf}_{1} f_{1}\left(t_{N}\right) C_{m_{1}}+\mathrm{rf}_{2} f_{2}\left(t_{N}\right) C_{m_{2}}+\cdots+\mathrm{rf}_{N} f_{N}\left(t_{N}\right) C_{m_{N}}=I_{\mathrm{T}}\left(t_{N}\right)
\end{aligned}
$$


sivity for each mass-to-charge ratio chronogram can be determined easily. Furthermore, the sample concentration can be obtained from the midpoint flux, which is sensitive only to changes of concentration and is independent of diffusivity. For more complex mixtures when the chronograms are each affected by several components and each component affects several chronograms, individual component concentrations can be obtained from the solution of a matrix of linear equations for each time and mass-to-charge ratio sampled.

\section{Acknowledgments}

We would like to thank Dr. Mark LaPack for his earlier work on membrane inlet mass spectrometry and his suggestions on this work. We also would like to acknowledge the partial financial support from Dow Chemical Company.

\section{References}

1. Bier, M. E.; Cooks, R. G.; Tou, J. C.; Westover, L. B. U.S Patent 4791 292, 1989.

2. Bier, M. E.; Cooks, R. G. Anal. Chem. 1987, 59, 597.
3. LaPack, M. A.; Tou, J. C.; Enke, C. G. Anal. Chem. 1991, 63, 1631.

4. Cameron, D.; Hemberger, P. H.; Alarid, J. E.; Lerbman, C. P.; Williams,. J. D. J. Am. Soc. Mass Spectrom. 1993, 4, 774.

5. Xu, C.; Patrick, J. S.; Cooks, R. G. Alnal. Che'n. 1995, 67, 724.

6. Tsai, G.-J. A.; Glen, D.; Syu, M. J.; Tsao, G. T.; I layward, M. J.; Kotiaho, T.; Cooks, R. G. Anal. Chem. 1991, 63, 2460.

7. Finlayson, B. A. Nonlinear Analysis in Chemical Engineering; McGraw-Hill International: New York, 1980; p 214.

8. Crank, J. The Mathe'matics of Diffision, 2 ed.; Clarendon Press: Oxford, 1975; p 47.

9. Crank, J.; Nicolson, P. Proc. Cambridge Phil. Soc. 1947, 43, 50.

10. LaPack, M. A. Ph.D. Thesis, Michigan State University, 1991.

11. Ben-Naim, A. Solvation Thermodymamics; Plenum Press: New York, 1987.

12. Karger, B. L.; Snyder, L. R. An Introduction to Separation Science; Wiley-Interscience: New York, 1973.

13. Lee, C. H. J. Appl. Polym. Sci. 1975, 19, 83.

14. Pasternak, R. A.; Schimscheimer, J. F.; Heller, J. I. Polym. Sci. 1970, 8, 467.

15. Koonin, S. E; Meredith, D. C. Computational Physics; Addison-Wesley: Reading, MA, 1970.

16. Press, W. H.; Teukolsky, S. A.; Vetterling, W. T. Numerical Recipes in C, 2 ed.; Cambridge University Press: New York, 1992.

17. Britz, D. Digital Simulation in Electrochemistry, 2nd (revised and extended) ed.; Springer-Verlag: Berlin, 1988; p 81. 